\title{
An Observational, Longitudinal Study of Cognition in Medical Cannabis Patients over the Course of 12 Months of Treatment: Preliminary Results
}

\author{
Kelly A. Sagar ${ }^{1,2,3}$, M. Kathryn Dahlgren ${ }^{1,2,3}$, Ashley M. Lambros ${ }^{1,2}$, Rosemary T. Smith ${ }^{1,2}$, Celine El-Abboud ${ }^{1,2}$ and \\ Staci A. Gruber ${ }^{1,2,3, *}$ (D) \\ ${ }^{1}$ Cognitive and Clinical Neuroimaging Core, McLean Hospital Imaging Center, 115 Mill St, Belmont, MA 02478, USA \\ ${ }^{2}$ Marijuana Investigations for Neuroscientific Discovery (MIND) Program, McLean Hospital Imaging Center, 115 Mill St, Belmont, MA 02478, USA \\ ${ }^{3}$ Department of Psychiatry, Harvard Medical School, 401 Park Dr, Boston, MA 02215, USA
}

(Received August 3, 2020; Final Revision November 17, 2020; AcCePted January 6, 2021)

\begin{abstract}
Objective: Cannabis use has increased dramatically across the country; however, few studies have assessed the long-term impact of medical cannabis (MC) use on cognition. Studies examining recreational cannabis users generally report cognitive decrements, particularly in those with adolescent onset. As MC patients differ from recreational consumers in motives for use, product selection, and age of onset, we assessed cognitive and clinical measures in well-characterized MC patients over 1 year. Based on previous findings, we hypothesized MC patients would not show decrements and might instead demonstrate improvements in executive function over time. Method: As part of an ongoing study, MC patients completed a baseline visit prior to initiating MC and evaluations following 3, 6, and 12 months of treatment. At each visit, patients completed a neurocognitive battery assessing executive function, verbal learning/memory, and clinical scales assessing mood, anxiety, and sleep. Exposure to delta-9-tetrahydrocannabinol (THC) and cannabidiol (CBD) was also quantified. Results: Relative to baseline, MC patients demonstrated significant improvements on measures of executive function and clinical state over the course of 12 months; verbal learning/ memory performance generally remained stable. Improved cognitive performance was not correlated with MC use; however, clinical improvement was associated with higher CBD use. Analyses suggest cognitive improvements were associated with clinical improvement. Conclusions: Study results extend previous pilot findings, indicating that MC patients may exhibit enhanced rather than impaired executive function over time. Future studies should examine distinctions between recreational and MC use to identify potential mechanisms related to cognitive changes and the role of clinical improvement.
\end{abstract}

Keywords: Medical cannabis, cognition, executive function, clinical state, cannabinoids, longitudinal

\section{INTRODUCTION}

Decades of research have examined the association between recreational cannabis use and cognitive outcomes; however, few studies have examined the relationship between cognitive function and medical cannabis (MC) use, defined as using cannabis specifically to treat symptoms of a medical condition. Although recreational and MC products are derived from the same plant species (Russo, 2007), inherent differences typically exist between those who use cannabis recreationally and those who use for medical purposes.

*Correspondence and reprint requests to: Staci A. Gruber, McLean Hospital, 115 Mill Street, Belmont, MA 02478, USA. E-mail: gruber@ mclean.harvard.edu
Recreational cannabis use often begins during adolescence (Substance Abuse and Mental Health Services Administration, 2019), a period marked by critical neurodevelopment (Casey, Galvan, \& Hare, 2005; Giedd et al., 1999; Gogtay et al., 2006; Houston, Herting, \& Sowell, 2014; Lebel $\&$ Deoni, 2018). Many studies assessing recreational cannabis users have demonstrated that earlier cannabis onset is related to poorer cognitive performance (Gruber \& Sagar, 2017; Lisdahl, Gilbart, Wright, \& Shollenbarger, 2013; Sagar \& Gruber, 2018), likely the result of cannabis exposure during this period of neurodevelopmental vulnerability (Casey et al., 2005; Giedd et al., 1999; Gogtay et al., 2006). As the endocannabinoid system impacts growth, differentiation, positioning, and connectivity among neurons, exposure to exogenous cannabinoids, specifically 
delta-9-tetrahydrocannabinol (THC), the primary intoxicating constituent of cannabis, may disrupt neural development, particularly during adolescence. In contrast, the majority of MC patients initiate MC use during adulthood (Gruber et al., 2018; Ilgen et al., 2013), when they are beyond vulnerable developmental periods; later initiation of MC is likely due to increased prevalence of chronic conditions as individuals age (Atella et al., 2019) and MC laws which generally apply to adults. Accordingly, recreational users and MC patients may experience different cognitive effects of cannabis given potential differences in age of onset of use.

In addition, recreational users and MC patients may have access to the same products, but recreational users typically choose products with considerable amounts of THC as they desire "high" or mood-altering effects (Wachtel, ElSohly, Ross, Ambre, \& de Wit, 2002; Zeiger et al., 2010). MC patients, whose primary goal is symptom alleviation (Nunberg, Kilmer, Pacula, \& Burgdorf, 2011), may also use products containing THC which can confer therapeutic effects such as analgesia (De Vita, Moskal, Maisto, \& Ansell, 2018), antiemesis, and appetite stimulation (Abrams, 2016; Walsh, Nelson, \& Mahmoud, 2003). However, they often want to avoid feeling intoxicated, and frequently seek products with varied cannabinoid profiles, particularly those containing cannabidiol (CBD), a non-intoxicating cannabinoid touted for its therapeutic potential.

Additional research studies, particularly clinical trials, are needed to determine the potential efficacy of CBD for various conditions (Black et al., 2019; NASEM, 2017). Although data are currently mixed, various factors including dosing and CBD product type (e.g., single, purified vs. full- or broad-spectrum) likely impact findings (Gallily, Yekhtin, \& Hanuš, 2015; Millar et al., 2019), and several studies have highlighted promising results (Bonaccorso, Ricciardi, Zangani, Chiappini, \& Schifano, 2019). Studies suggest CBD may mitigate or decrease negative effects often associated with THC exposure, including cognitive decrements, as noted in a study examining the effects of smoked cannabis with higher versus lower amounts of CBD (Morgan, Schafer, Freeman, \& Curran, 2010), and a study in which frequent cannabis users were administered $200 \mathrm{mg}$ oral CBD for 10 weeks (Solowij et al., 2018). Although some studies found THC induces psychotic-like symptoms or anxiogenic effects at high doses (Hunault et al., 2014; Martin-Santos et al., 2012), these effects may be mitigated by $\mathrm{CBD}$, as demonstrated in a study assessing the impact of smoked cannabis with varied cannabinoid levels (Schubart et al., 2011). Additionally, Bergamaschi et al. (2011) reported that pre-administration of $600 \mathrm{mg}$ oral CBD prior to a Simulated Public Speaking Test reduced anxiety, discomfort, and cognitive impairment relative to a placebo group. Similarly, Crippa et al. (2011) reported reduced anxiety and altered limbic activity following oral administration of $400 \mathrm{mg}$ CBD (Crippa et al., 2011). Overall, THC and CBD have been shown to have opposing neural effects, particularly in regions rich in cannabinoid receptors (Lorenzetti, Solowij, \& Yucel, 2016).
It is possible that if MC treatment reduces physical or psychological symptoms, cognitive function may actually improve as patients feel better. For example, chronic pain, the most common indication for MC use (Park \& Wu, 2017), has been shown to adversely impact cognitive performance, specifically tasks requiring attention and executive function (Moriarty, McGuire, \& Finn, 2011). Sleep has also been associated with cognitive function; good sleep quality promotes better cognitive function and protects against age-related cognitive decline and dementia (Minakawa, Wada, \& Nagai, 2019; Scullin \& Bliwise, 2015; Shi et al., 2018). Additionally, studies note that anxiety, another common indication for MC (Grella, Rodriguez, \& Kim, 2014), interferes with attention and executive function (Vytal, Cornwell, Letkiewicz, Arkin, \& Grillon, 2013). Certain cannabinoids, particularly CBD, appear to have anxiolytic properties, demonstrated by acute administration studies in healthy volunteers (Zuardi, Cosme, Graeff, \& Guimaraes, 1993) and individuals with anxiety disorders (Bergamaschi et al., 2011). A large-case series also reported a $79 \%$ improvement in retrospective anxiety ratings after 1 month of CBD treatment (Shannon, Lewis, Lee, \& Hughes, 2019). MC treatment could therefore be associated with better concentration and enhanced cognitive performance if primary medical symptoms are reduced.

Given inherent differences between medical and recreational cannabis use, it is likely that MC patients will not exhibit the same pattern of decrements on neuropsychological measures traditionally observed in young, heavy recreational users (Crean, Crane, \& Mason, 2011; Jacobus \& Tapert, 2014; Lisdahl, Wright, Kirchner-Medina, Maple, \& Shollenbarger, 2014). Interestingly, in the first study to directly assess patients using "real world" MC products pre- versus post-MC treatment, we collected pilot data in 11 patients at baseline (prior to initiating $\mathrm{MC}$ use), and following 3 months of regular MC treatment (Gruber et al., 2016). In contrast to executive function decrements typically observed in recreational cannabis users, MC patients exhibited improved performance on the Stroop Color Word Test and Trail Making Test, reflected by faster response time without loss of accuracy. Further, MC patients experienced cognitive improvements in the context of moderate improvements on measures of depression, sleep, and quality of life.

Additionally, Olla et al (2019) recently examined the short-term effects of THC in MC patients at baseline (not intoxicated), immediately after using high THC (20\%) cannabis products, and several hours later. Although the authors hypothesized poorer performance during intoxication, findings revealed stable or improved performance across several cognitive domains. Of note, findings may have been significantly impacted by practice effects, as alternate test versions were not used, and only a short period of time elapsed between assessments. However, as several studies report poorer cognitive performance following acute cannabis administration (Desrosiers, Ramaekers, Chauchard, Gorelick, \& Huestis, 2015; Hart et al., 2010; Hart, van Gorp, Haney, Foltin, \& Fischman, 2001), results from Olla and colleagues provide further evidence that patients 
using cannabis medically may not experience the same decrements often observed in recreational consumers.

In order to more thoroughly examine the long-term impact of MC treatment on cognition, the current, ongoing study expanded our pilot investigation (Gruber et al., 2016), utilizing a longitudinal design where MC patients completed baseline cognitive and clinical assessments prior to initiation of $\mathrm{MC}$ use and were reassessed at multiple time points following initiation of MC treatment (3, 6, and 12 months). Information regarding $\mathrm{MC}$ treatment regimens, including frequency of use and exposure to THC and CBD were also quantified to determine if $\mathrm{MC}$ use variables contributed to cognitive and clinical changes.

\section{Hypotheses}

Based on pilot work as well as the fact that MC patients are often older and may choose non-intoxicating products or those with varied cannabinoid profiles, we hypothesized that MC patients would not demonstrate decrements and might instead exhibit improvements in cognitive function after initiating regular MC use for up to 1 year. Further, we predicted that MC patients would report improved clinical ratings following $\mathrm{MC}$ treatment. Additional analyses examined potential relationships between changes in cognitive function and self-reported clinical ratings as well as measures of cannabinoid use (e.g., frequency of use, THC, and/or CBD exposure).

\section{METHODS}

This research was completed in accordance with the Declaration of Helsinki.

\section{Participants}

To date, $54 \mathrm{MC}$ patients have been successfully enrolled and completed at least one follow-up assessment $(3,6$, and/or 12 months of MC use). Of the $54 \mathrm{MC}$ patients included in the current analyses, 51 completed a 3-month follow-up, 44 completed a 6-month follow-up, and 32 returned after 12 months (ns $=50,41$, and 29, respectively, for completion of neuropsychological assessments). More specifically, 27 patients completed all four visits, and five missed an interim visit(s). Ten participants remain enrolled but are awaiting their next follow-up timepoint. Only 12 patients (22\%) were discontinued or withdrew from the study because they either stopped MC use $(n=3)$ or were lost to follow-up $(n=9)$. As not all patients completed all four timepoints, we compared baseline data between completers and noncompleters to assess the potential impact of missing data. Completers and non-completers did not differ significantly on any variable with the exception of Trails B errors ( $p=.03$ ); however, both groups made less than one error. Therefore, missing data should be considered missing completely at random (MCAR) and unlikely to influence results.
Participants were recruited for the study via ads targeting individuals interested in using MC; ads were posted on our research volunteer portal, social media, and at MC certification centers. Study staff did not facilitate MC certification. To qualify for entry, participants had to be 21 or older and have either a valid certification for MC or report a desire to use hemp-based products, which do not currently require certification, in an attempt to enroll only those interested in using cannabis for medical purposes. Patients could plan to use MC for a variety of indications, including chronic pain, anxiety, mood, sleep, or other medical/psychiatric conditions. MC patients must not have begun regular MC treatment or endorsed recent recreational cannabis use prior to baseline assessments. Specifically, participants were either required to be cannabis naïve ( $\leq 15$ lifetime uses) or, if they reported a history of cannabis use, they could not have regular recreational use $(>1 \mathrm{x} / \mathrm{month})$ within the last year to limit effects of recent exposure. To help confirm this entry criterion, all patients were required to test negative for urinary THC metabolites at baseline. In addition, all patients completed the two-factor Wechsler Abbreviated Scale of Intelligence (WASI; (Wechsler, 1999) at baseline and were required to have an IQ of $\geq 75$.

\section{Study Design}

Upon arrival, study procedures were explained, and patients were required to read and sign an informed consent form approved by the Partners Healthcare Institutional Review Board. Prior to initiating MC treatment, all patients completed a neurocognitive battery and measures of clinical state and sleep quality. Following 3, 6, and 12 months of MC treatment, patients returned to repeat study measures. The neuropsychological test battery was designed to assess executive function and memory, as studies of recreational users have shown these domains to be most vulnerable to cannabis use (Sagar \& Gruber, 2018). To examine various aspects of executive function, patients completed the Stroop Color Word Test (MacLeod, 1991), Trail Making Test (Lezak, Howieson, Bigler, \& Tranel, 2012), computerized Wisconsin Card Sorting Test (WCST-64; Berg, 1948; Heaton \& PAR Staff; Lezak et al., 2012), Letter-Number Sequencing (LNS) subtest of the Wechsler Adult Intelligence Scale-Revised (WAIS-R; Wechsler, 1987), and the Controlled Oral Word Association Test (COWAT; Strauss, Sherman, \& Spreen, 2006). Verbal learning and memory were assessed using the Rey Auditory Verbal Learning Test (RAVLT; Schmidt, 2016). To limit practice effects, alternate test forms were used at each follow-up visit for all tasks except Stroop and WCST.

To examine potential clinical changes related to MC use which could impact cognitive performance, various aspects of clinical state were assessed using the Profile of Mood State (POMS; Pollock, Cho, Reker, \& Volavka, 1979), which provides a score reflecting Total Mood Disturbance (TMD); the Beck Depression Inventory (BDI; Beck, Ward, Mendelson, Mock, \& Erbaugh, 1961); the Beck Anxiety 
Inventory (BAI; Beck \& Steer, 1990); and the State Trait Anxiety Index (STAI; Spielberger, Gorsuch, Lushene, Vagg, \& Jacobs, 1983), which provides subscores for state (how one currently feels) and trait anxiety (how one generally feels). For these scales, higher scores are indicative of increased symptomatology. MC patients also completed the Pittsburgh Sleep Quality Index (PSQI; Buysse, Reynolds, Monk, Berman, \& Kupfer, 1989); higher scores indicate poorer sleep.

Upon completion of baseline procedures, MC patients were provided with drug diaries to track MC use. Diaries recorded product information, route of administration, frequency, and amount of MC used. Between visits, patients were contacted monthly by phone to corroborate diary entries using a modified timeline follow-back procedure (TLFB) (Robinson, Sobell, Sobell, \& Leo, 2014), which utilizes cannabis-specific follow-up queries. During phone check-ins, discrepancies with diary information were rectified using additional queries to ensure accurate information was recorded.

To gather information about cannabinoid content within each product, each patient was asked to provide a sample of their most frequently used MC product(s) for cannabinoid constituent profiling, completed by an outside laboratory (ProVerde Laboratories, Inc.). Although reports provided information on 12 cannabinoids, specific focus was placed on THC and CBD. When patients were unable to supply a sample for analysis, constituent information was gathered from certificates of analyses (COAs) from manufacturers/ dispensaries, or from product labels when COAs were unavailable. Using constituent information along with use data from diary entries and/or TLFB queries, a standard metric of cannabinoid exposure, measured in $\mathrm{mg}$ of $\mathrm{THC}$ and CBD used per week, was calculated for each patient at each follow-up visit.

\section{Statistical Analyses}

For all analyses, raw scores were utilized for cognitive performance data and self-report ratings, as methods for standardization were not uniform across assessments. Repeated Measures Analyses of Variance (rmANOVAs, 2-tailed) were used to assess within-subject changes for cognitive and clinical variables. As changes from baseline were the contrasts of interest, analyses compared data at each follow-up visit relative to baseline ratings (i.e., baseline vs. 3 months, baseline vs. 6 months, and baseline vs. 12 months); these methods maximized study sample size and statistical power. Results from each of these analyses are reported with effect sizes (partial eta squared) and 95\% confidence intervals. Expected power for all contrasts was calculated as $\geq 86.96 \%$ for medium effect sizes and $\geq 97.23 \%$ for large effect sizes.

For cognitive and clinical measures demonstrating consistent, statistically significant patterns of change at follow-up visits relative to baseline, bivariate correlation analyses (Pearson's $r$, 2-tailed) were used to assess the relationship between changes in cognitive performance and clinical ratings as well as the association between these variables and cannabinoid exposure (frequency, mg of THC/week, $\mathrm{CBD} /$ week). For these correlations, difference scores comparing baseline to 3 months were utilized in order to maximize sample sizes and statistical power.

\section{RESULTS}

\section{Demographics}

To date, $54 \mathrm{MC}$ patients (20M, $34 \mathrm{~F})$ between the ages of 23-78 completed baseline assessments and returned for at least one follow-up visit (Table 1). Of the 54 patients in this sample, 26 were cannabis naive ( $\leq 15$ lifetime uses), and 28 reported a history of past recreational cannabis use with at least 12 months of abstinence from regular use (defined as $>1 \mathrm{x} / \mathrm{month}$ ). See Table 1 for information regarding initiation/cessation and duration of abstinence from recreational use. All participants tested negative for THC metabolites at baseline.

Patients used MC to treat a variety of symptoms and conditions, including pain $(n=36)$, anxiety or PTSD $(n=31)$, sleep $(n=22)$, mood $(n=14)$, attention $(n=4)$, and other medical conditions $(n=4) ; 36$ patients reported using MC to treat more than one condition. Over the course of the study, patients reported using MC 9-11 times/week on average. Interestingly, cannabinoid exposure calculations revealed that overall, THC exposure (mg/week) was notably lower than CBD exposure (mg/week) at each visit; this difference was only statistically significant for MC use after 6 months of treatment $(F(1,31)=7.73, p=.01)$.

\section{Cognition}

Over the course of 12 months of MC treatment, patients exhibited significant changes on several measures of executive functioning (see Table 2). On the Stroop Interference condition, MC patients exhibited significantly faster times at all follow-up visits relative to baseline (all $p s<.01$ ), suggesting improved inhibitory processing. Overall, MC patients exhibited high levels of accuracy (Range: 97.86-98.76\%) across visits. Although a statistically significant reduction in accuracy was noted at 3 months relative to baseline $(p<.01)$, given the high levels of accuracy attained and very small change from baseline to 3 months $(<1 \%)$, this does not appear clinically significant. Further, as this pattern was not observed at later visits, results suggest that improvements in Stroop completion times did not come at the expense of lower task accuracy.

On the Trail Making Test Part B, MC patients demonstrated significantly faster times between baseline and 3 months $(p=.04)$, but no significant differences were observed after 6 or 12 months compared to baseline. Although significantly higher error rates were observed after 6 and 12 months of MC use ( $p=.02$ and .03 , respectively), errors remained quite low at all visits $(<1$ error), suggesting differences in task performance were not clinically significant. 
Table 1. MC patient demographics \& medical cannabis/cannabinoid use

\begin{tabular}{|c|c|}
\hline Demographics* & $M(S D)$ or $n(\%)$ \\
\hline Sex & $\begin{array}{c}20 \text { Male }(37.04 \%) \\
34 \text { Female }(62.96 \%)\end{array}$ \\
\hline Race & $\begin{array}{c}48 \text { White }(88.89 \%) \\
5 \text { Non-White }(9.26 \%) \\
1 \text { Prefer Not to Answer }(1.85 \%)\end{array}$ \\
\hline Age & $49.17(16.45)$ \\
\hline Education & $16.57(1.99)$ \\
\hline IQ & $121.02(7.54)$ \\
\hline \multicolumn{2}{|l|}{ History of Recreational Cannabis Use } \\
\hline Previous Recreational Use & $\begin{array}{c}6 \text { Never Tried }(11.11 \%) \\
20 \text { No Regular Use }{ }^{\dagger}(37.04 \%) \\
16 \text { Past Light Use }{ }^{\ddagger}(29.63 \%) \\
12 \text { Past Frequent Use }{ }^{\S}(22.22 \%)\end{array}$ \\
\hline Age Regular Recreational Use Started & $18.70(5.45)$ \\
\hline Age Regular Recreational Use Ended & $24.00(6.79)$ \\
\hline Duration of Abstinence from Regular Use (years) & $23.57(14.20)$ \\
\hline \multicolumn{2}{|l|}{ Medical Cannabis (MC) Uses/Week } \\
\hline $\begin{array}{l}3 \text { Months } \\
6 \text { Months }^{\text {II }} \\
12 \text { Months }^{* *}\end{array}$ & $\begin{array}{r}9.29(6.28) \\
10.20(8.25) \\
11.19(7.86)\end{array}$ \\
\hline \multicolumn{2}{|l|}{ MC: THC mg/week } \\
\hline $\begin{array}{l}3 \text { Months }^{\dagger \dagger} \\
6 \text { Months }^{\dagger \dagger} \\
12 \text { Months }^{\S \S}\end{array}$ & $\begin{array}{l}63.97(184.18) \\
41.89(78.78) \\
35.99(48.86)\end{array}$ \\
\hline \multicolumn{2}{|l|}{ MC: CBD mg/week } \\
\hline $\begin{array}{l}3 \text { Months }^{\dagger \dagger} \\
6 \text { Months }^{\dagger \neq} \\
12 \text { Months }^{\S \S}\end{array}$ & $\begin{array}{l}153.90(287.79) \\
201.64(321.38) \\
113.50(251.47)\end{array}$ \\
\hline \multicolumn{2}{|l|}{ Routes of MC Administration $\|$} \\
\hline $\begin{array}{l}\text { Smoke } \\
\text { Vape } \\
\text { Oromucosal (Oil, Tincture, Solution) } \\
\text { Oral (Edible, Tablet, Capsule) } \\
\text { Cutaneous (Lotion, Salve) } \\
\text { Transdermal } \\
\text { Transmucosal (Suppository) }\end{array}$ & $\begin{aligned} 13 & (55.56 \%) \\
27 & (50.00 \%) \\
33 & (61.11 \%) \\
22 & (40.74 \%) \\
5 & (9.26 \%) \\
0 & (0.0 \%) \\
0 & (0.0 \%)\end{aligned}$ \\
\hline
\end{tabular}

THC = delta-9-tetrahydrocannabinol; $\mathrm{CBD}=$ cannabidiol.

$* n=54$, unless otherwise noted.

${ }^{\dagger}$ No regular use was defined as $<1 \mathrm{x} /$ month and a maximum of 15 lifetime uses.

$¥$ Light use was defined as regular use of at least $1 \mathrm{x} /$ month but $<2 \mathrm{x} /$ week.

\& Frequent use was defined as regular use of at least $2 \mathrm{x} / \mathrm{week}$.

I $n=50$.

$\mathbb{I} n=44$.

$* * n=31$.

部 $n=38$.

$\$ n=32$.

$\S \S n=24$.

II Participants could report multiple modes of use.

On the WCST, MC patients generally exhibited improved performance following initiation of MC treatment. Total categories achieved was higher at all follow-up visits relative to baseline, although this increase was only significant after 6 months of treatment $(p=.02)$. In addition, perseverative errors generally decreased across visits; however, this was only statistically significant following 12 months of $\mathrm{MC}$ treatment $(p=.01)$.

On the LNS, statistically significant improvements were detected at all three follow-up visits relative to baseline (all $p \mathrm{~s} \leq .01$ ). Performance on the COWAT remained relatively stable after 3 and 12 months of treatment, but statistically 
Table 2. Changes in cognitive performance over the course of 3, 6, and 12 months of MC treatment

\begin{tabular}{|c|c|c|c|c|c|c|c|c|c|c|c|c|}
\hline \multirow[b]{2}{*}{ Task Variable } & \multirow{2}{*}{$\begin{array}{c}\begin{array}{c}\text { Baseline } \\
(n=50)\end{array} \\
M(S D)\end{array}$} & \multirow{2}{*}{$\begin{array}{c}\begin{array}{c}3 \text { Months } \\
(n=50)\end{array} \\
M(S D)\end{array}$} & \multicolumn{2}{|l|}{ ANOVA* $^{*}$} & \multirow{2}{*}{$\begin{array}{c}\begin{array}{c}\text { Baseline } \\
(n=41)\end{array} \\
M(S D)\end{array}$} & \multirow{2}{*}{$\begin{array}{c}6 \text { Months } \\
(n=41)\end{array}$} & \multicolumn{2}{|l|}{ ANOVA $\dagger$} & \multirow{2}{*}{$\begin{array}{c}\text { Baseline } \\
(n=29)\end{array}$} & \multirow{2}{*}{$\begin{array}{c}12 \text { Months } \\
(n=29)\end{array}$} & \multicolumn{2}{|l|}{ ANOVA $\neq$} \\
\hline & & & $F(95 \% \mathrm{CI})$ & $p\left(\eta^{2}\right)$ & & & $F(95 \% \mathrm{CI})$ & $p\left(\eta^{2}\right)$ & & & $F(95 \% C I)$ & $p\left(\eta^{2}\right)$ \\
\hline \multicolumn{13}{|l|}{ Executive Function } \\
\hline $\begin{array}{l}\text { Stroop Interference } \\
\text { Time (s) }\end{array}$ & $105.44(25.27)$ & $97.72(24.38)$ & $26.92(4.73,10.71)$ & $<.01(.36)$ & $105.34(24.83)$ & $93.07(21.04)$ & $49.89(8.76,15.78)$ & $<.01(.56)$ & $103.76(22.67)$ & $90.59(17.94)$ & $35.54(8.65,17.70)$ & $<.01(.56)$ \\
\hline $\begin{array}{c}\text { Stroop Interference } \\
\text { Accuracy }(\%)\end{array}$ & $98.56(1.36)$ & $97.86(1.98)$ & $9.96(0.25,1.15)$ & $<.01(.17)$ & $98.56(1.32)$ & $98.61(1.30)$ & $0.05(-0.51,0.41)$ & $.83(<.01)$ & $98.76(1.21)$ & $98.55(1.76)$ & $0.39(-0.47,0.88)$ & $.54(.01)$ \\
\hline Trails B Time (s) & $60.70(18.02)$ & 56.06 & $4.55(0.27,9.01)$ & $.04(.09)$ & $60.93(18.27)$ & $66.17(22.20)$ & $3.53(-10.89,0.40)$ & $.07(.08)$ & $56.90(14.85)$ & $61.62(22.16)$ & $1.95(-11.66,2.21)$ & $.17(.07)$ \\
\hline Trails B Errors & $0.30(0.54)$ & $0.26(0.53)$ & $0.14(-0.18,0.26)$ & $.71(<.01)$ & $0.27(0.55)$ & $0.66(0.91)$ & $6.28(0.08,0.71)$ & $.02(.14)$ & $0.14(0.44)$ & $0.62(1.02)$ & $5.37(0.06,0.91)$ & $.03(.16)$ \\
\hline WCST Categories & $3.04(1.37)$ & $3.32(1.36)$ & $3.09(-0.60,0.04)$ & $.09(.06)$ & $3.29(1.25)$ & $3.76(1.16)$ & $6.27(-0.84,-0.09)$ & $.02(.14)$ & $3.38(1.35)$ & $3.69(1.44)$ & $1.77(-0.79,0.17)$ & $.19(.06)$ \\
\hline $\begin{array}{l}\text { WCST Perseverative } \\
\text { Errors }\end{array}$ & $8.62(4.30)$ & $8.18(5.24)$ & $0.44(-0.89,1.77)$ & $.51(.01)$ & $8.63(4.38)$ & $7.24(3.83)$ & $2.75(-0.31,3.09)$ & $.11(.06)$ & $8.21(4.44)$ & $6.55(2.90)$ & $6.98(0.37,2.94)$ & $.01(.20)$ \\
\hline LNS & $12.00(2.59)$ & $13.02(2.96)$ & $12.44(-1.60,-0.44)$ & $<.01(.20)$ & $12.34(2.72)$ & $13.24(3.10)$ & $8.16(-1.54,-0.26)$ & $.01(.17)$ & $12.03(2.83)$ & $13.45(3.44)$ & $11.19(-2.28,-0.55)$ & $<.01$ \\
\hline COWAT & $49.52(13.78)$ & $48.92(13.51)$ & $0.37(-1.37,2.57)$ & $.54(.01)$ & $51.59(14.42)$ & $54.89(14.14)$ & $7.74(-5.68,-0.90)$ & $.01(.16)$ & $50.31(13.95)$ & $51.72(12.72)$ & $0.91(-4.44,1.62)$ & $.35(.03)$ \\
\hline \multicolumn{13}{|c|}{ Verbal Learning and Memory } \\
\hline $\begin{array}{l}\text { RAVLT Trials 1-5 } \\
\text { Correct }\end{array}$ & $50.86(8.78)$ & $49.84(8.47)$ & $1.04(-0.99,3.03)$ & $.31(.02)$ & $51.54(9.49)$ & $53.73(7.80)$ & $3.87(-4.45,0.06)$ & $.06(.09)$ & $52.41(8.74)$ & $52.83(8.55)$ & $0.08(-3.35,2.53)$ & $.78(<.01)$ \\
\hline $\begin{array}{l}\text { RAVLT Short } \\
\text { Delay }\end{array}$ & $10.76(2.98)$ & $10.36(2.74)$ & $0.95(-0.43,1.23)$ & $.34(.02)$ & $10.78(3.19)$ & $11.24(2.68)$ & $1.48(-1.23,0.31)$ & $.23(.04)$ & $11.34(2.86)$ & $11.31(2.63)$ & $0.01(-0.84,0.91)$ & $.94(<.01)$ \\
\hline RAVLT Long Delay & $10.80(3.08)$ & $10.22(2.96)$ & $2.66(-0.14,1.30)$ & $.11(.05)$ & $10.80(3.39)$ & $11.00(2.74)$ & $0.28(-0.94,0.55)$ & $.60(.01)$ & $11.38(2.81)$ & $10.66(3.09)$ & $4.54(0.03,1.42)$ & $.04(.14)$ \\
\hline
\end{tabular}

COWAT = Controlled Oral Word Association Test; LNS = Letter-Number Sequencing; MC = medical cannabis; RAVLT = Rey Auditory Verbal Learning Test; WCST = Wisconsin Card Sorting Test

COWAT $=$ Controlled Oral Word Associc
Significant values $(p \leq .05)$ are bolded.

*Degrees of freedom $(\mathrm{df})=1,49$.

$\dagger \mathrm{df}=1,40$.

${ }^{\ddagger} \mathrm{df}=1,28$. 
significant improvements were noted following 6 months of treatment $(p=.01)$.

On the RAVLT, verbal learning and memory performance were generally maintained during follow-up visits relative to baseline, as few statistically significant changes were noted. While patients recalled fewer words on the long delay condition after 12 months of treatment relative to baseline ( $p=.04)$, closer analysis revealed that MC patients remembered approximately 11 total words at both visits (baseline $=11.38 ; 12$ months $=10.66$ ), suggesting that while this difference is statistically significant, it does not appear to be clinically significant.

\section{CLINICAL RATINGS: MOOD, ANXIETY, AND SLEEP}

Clinical ratings are reported in Table 3. On the POMS, patients exhibited significantly decreased total mood disturbance (TMD) at each follow-up visit relative to baseline (all $p s \leq .05$ ). Further, BDI scores indicated significant reductions in self-reported symptoms of depression across all follow-up visits relative to baseline (all $p$ s $<.01$ ). Additionally, anxiety ratings generally decreased over time. BAI scores were significantly lower after 6 and 12 months of MC treatment (both $p=.02)$. On the STAI, trait anxiety significantly decreased at follow-up visits relative to baseline (all $p s<.01$ ), while state anxiety generally showed a pattern of decreased anxiety; this reached significance for only the baseline to 6-month comparison $(p<.01)$. On the PSQI, patients reported better sleep quality at all follow-up visits after initiating MC treatment (all $p \mathrm{~s}<.01$ ).

\section{Correlations: MC Use versus Cognitive Variables}

Correlation analyses explored potential relationships between MC use variables (MC use episodes/week, THC $\mathrm{mg} /$ week, CBD mg/week) and select key cognitive variables demonstrating statistically significant changes between baseline and 3 months: Stroop Interference time, Trails B time, and LNS total. After controlling for outliers, no significant relationships were observed between any MC use variable and any cognitive performance variable.

\section{Correlations: MC Use versus Clinical Variables}

Correlation analyses examining relationships between MC use and clinical variables (POMS TMD, BDI, STAI trait anxiety, and PSQI) revealed that improvements in mood and anxiety were significantly associated with higher CBD exposure (mg/week). Specifically, improvement on POMS TMD $(r(36)=.737, p<.001)$, BDI $(r(36)=.521$, $p=.001)$, and STAI trait anxiety $(r(36)=.634, p<.001)$ correlated with increased CBD use. Additionally, improved trait anxiety on the STAI was significantly correlated with greater MC use episodes per week $(r(48)=.327$, $p=.021)$. Importantly, THC exposure was not associated with any clinical variables, and no other relationships were observed between MC use and clinical changes.

\section{Correlations: Cognitive versus Clinical Variables}

As exposure to individual cannabinoids did not directly impact cognitive function, correlations assessed whether the observed cognitive improvements may be related to clinical improvements, suggestive of an indirect effect of MC use on cognition. Results revealed that faster Stroop Interference times were significantly associated with clinical improvement: POMS TMD $(r(48)=.411, p=.003)$, BDI $(r(48)=.437, p=.002)$, STAI trait anxiety $(r(48)=$ $.487, p<.001)$, and PSQI $(r(45)=.288, p=.049)$. No significant relationships were observed between any other cognitive and clinical variables.

\section{DISCUSSION}

In contrast to studies reporting decrements in cognitive performance among recreational cannabis consumers, particularly those with adolescent onset (Crean et al., 2011; Lisdahl et al., 2014; Sagar \& Gruber, 2018), data from the current investigation suggest that 3 to 12 months of MC use does not appear to be associated with poorer cognitive performance. These findings extend pilot findings which indicated improvement on some measures of executive function following 3 months of MC treatment (Gruber et al., 2016). The current longitudinal, observational study examined a larger sample of MC patients over a longer time course and found that MC patients demonstrated improvements on several executive tasks. Specifically, over time, patients exhibited faster time on the Stroop Interference condition while maintaining high levels of accuracy. Performance on the Trail Making Test suggested faster psychomotor speed, but more variable performance in terms of cognitive flexibility and set-shifting across visits. On the computerized version of the WCST, MC patients achieved more categories while making fewer perseverative errors. MC patients generally exhibited improved performance on the LNS at all follow-up visits, and phonemic fluency remained relatively stable with transient evidence of improvement over time. Interestingly, these improvements may not extend to measures of verbal memory; although verbal learning performance remained stable over time, some evidence for slightly decreased verbal memory following a long delay after 12 months of MC treatment emerged. While this finding was statistically significant, it is unlikely that there is clinical significance between recalling 11.38 words compared to 10.66 words. These results stand in contrast to findings in recreational cannabis users which frequently note decrements on measures of verbal learning and memory (Lisdahl et al., 2014; Sagar \& Gruber, 2018; Schwartz, Gruenewald, Klitzner, \& Fedio, 1989; Solowij et al., 2011).

Few studies have examined cognitive performance in those using cannabis for medical purposes. A recent 12-month longitudinal study utilized a single self-report measure of cognition, the Cognitive Failures Questionnaire, to examine individuals using cannabis to self-medicate for chronic medical conditions; although no improvements were 
Table 3. Changes in self-reported mood, anxiety, and sleep over the course of 3, 6, and 12 months of MC treatment

\begin{tabular}{|c|c|c|c|c|c|c|c|c|c|c|c|c|}
\hline \multirow[b]{2}{*}{ Scale } & \multirow{2}{*}{$\begin{array}{c}\begin{array}{c}\text { Baseline } \\
(n=51)\end{array} \\
M(S D)\end{array}$} & \multirow{2}{*}{$\begin{array}{c}3 \text { Months } \\
(n=51)\end{array}$} & \multicolumn{2}{|l|}{ ANOVA* } & \multirow{2}{*}{$\begin{array}{c}\begin{array}{c}\text { Baseline } \\
(n=44)\end{array} \\
M(S D)\end{array}$} & \multirow{2}{*}{$\begin{array}{l}6 \text { Months } \\
(n=44)\end{array}$} & \multicolumn{2}{|l|}{ ANOVA $\dagger$} & \multirow{2}{*}{$\begin{array}{c}\begin{array}{c}\text { Baseline } \\
(n=32)\end{array} \\
M(S D)\end{array}$} & \multirow{2}{*}{$\begin{array}{c}\begin{array}{c}12 \text { Months } \\
(n=32)\end{array} \\
M(S D)\end{array}$} & \multicolumn{2}{|l|}{ ANOVA } \\
\hline & & & $F(95 \% \mathrm{CI})$ & $p\left(\eta^{2}\right)$ & & & $F(95 \% \mathrm{CI})$ & $p\left(\eta^{2}\right)$ & & & $F(95 \% \mathrm{CI})$ & $p\left(\eta^{2}\right)$ \\
\hline \multicolumn{13}{|c|}{ Profile of Mood States (POMS) } \\
\hline $\begin{array}{l}\text { Total Mood } \\
\text { Disturbance } \\
\text { (TMD) }\end{array}$ & $34.12(42.14)$ & $26.45(40.55)$ & $3.99(-0.05,15.38)$ & $.05(.07)$ & $38.36(42.66)$ & $22.75(34.97)$ & $9.88(5.60,25.63)$ & $<.01(.19)$ & $30.78(37.80)$ & $18.88(27.20)$ & $5.80(0.46,21.94)$ & $.02(.16)$ \\
\hline \multicolumn{13}{|c|}{ Beck Depression Inventory (BDI) } \\
\hline BDI Total & $12.49(10.21)$ & $8.24(9.89)$ & $32.98(2.77,5.74)$ & $<.01(.40)$ & $13.57(10.09)$ & $6.73(6.86)$ & $27.33(4.20,9.48)$ & $<.01(.39)$ & $11.34(8.32)$ & $5.81(4.86)$ & $17.58(2.558 .25)$ & $<.01(.36)$ \\
\hline \multicolumn{13}{|c|}{ Beck Anxiety Inventory (BAI) } \\
\hline BAI Total & $10.61(9.56)$ & $8.45(9.06)$ & $2.93(-0.38,4.69)$ & $.09(.06)$ & $11.39(9.59)$ & $8.55(8.51)$ & $6.22(0.54,5.14)$ & $.02(.13)$ & $9.03(8.57)$ & $6.09(5.93)$ & $6.57(0.60,5.28)$ & $.02(.14)$ \\
\hline \multicolumn{13}{|c|}{ State-Trait Anxiety Inventory (STAI) } \\
\hline State Anxiety & $35.00(10.58)$ & $33.33(9.37)$ & $1.82(-0.82,4.15)$ & $.18(.04)$ & $36.09(10.67)$ & $31.75(8.16)$ & $12.11(1.83,6.86)$ & $<.01(.22)$ & $33.60(9.28)$ & $31.59(8.57)$ & $1.61(-1.22,5.22)$ & $.21(.05)$ \\
\hline Trait Anxiety & $42.53(14.32)$ & $39.04(13.13)$ & $10.72(1.35,5.63)$ & $<.01(.18)$ & $44.11(14.25)$ & $38.84(10.93)$ & $15.26(2.55,8.00)$ & $<.01(.26)$ & $41.31(13.25)$ & $36.88(11.73)$ & $12.85(1.91,6.96)$ & $<.01(.29)$ \\
\hline \multicolumn{13}{|c|}{ Pittsburgh Sleep Quality Index (PSQI) } \\
\hline PSQI Total & $8.96(4.32)$ & $7.13(3.59)$ & $11.21(0.73,2.94)$ & $<.01(.19)$ & $8.93(4.10)$ & $6.95(3.81)$ & $11.49(0.80,3.15)$ & $<.01(.22)$ & $8.52(4.27)$ & $6.24(3.27)$ & $20.24(1.24,3.31)$ & $<.01(.43)$ \\
\hline
\end{tabular}

$\mathrm{MC}=$ medical cannabis.

Significant values $(p \leq .05)$ are bolded.

$*$ Degrees of freedom $(\mathrm{df})=1,50$ for all scales except PSQI where $\mathrm{df}=1,47$

$\dagger \mathrm{df}=1,43$ for all scales except PSQI where $\mathrm{df}=1,40$.

$¥ \mathrm{df}=1,31$ except PSQI where $\mathrm{df}=1,28$. 
reported over time, no evidence of cognitive deterioration was reported over 12 months (Bouso et al., 2020). However, in contrast to the current investigation, study participants were not cannabis-naïve at baseline, which likely impacted baseline performance. As data from the current study suggest that improvement often occurs within 3 months of initiating treatment, cognitive improvements occurring in the early stages of MC initiation may not have been detected in the Bouso et al. (2020) sample, given no cannabis-naïve baseline assessment.

Importantly, improvements in cognitive performance in the current study appear to occur in the context of significant improvements on measures of mood, anxiety, and sleep. These findings are supported by retrospective reports of clinical improvements secondary to $\mathrm{MC}$ use among various patient populations. For example, a survey study of California residents found that of the $5 \%$ who reported having tried MC, $92 \%$ reported MC helped treat a serious medical condition (Ryan-Ibarra, Induni, \& Ewing, 2015). Another study of MC patients in Arizona reported that among those endorsing symptoms of anxiety, $83 \%$ reported "a lot or almost complete relief" from anxiety when using MC (Troutt \& DiDonato, 2015). In addition to these surveys, acute administration studies, observational studies, a handful of clinical trials, and several reviews have reported improvements in medical and psychiatric symptoms secondary to medical cannabis or cannabinoid use across a range of conditions and symptoms, including chronic pain (NASEM, 2017; Pawasarat et al., 2020; Poli, Crestani, Salvadori, Valenti, \& Sannino, 2018), anxiety (Bergamaschi et al., 2011; Masataka, 2019; Shannon et al., 2019; Zuardi et al., 1993; Zuardi, Shirakawa, Finkelfarb, \& Karniol, 1982), and sleep (Kuhathasan et al., 2019).

Several factors likely contribute to findings in the current study, the first to directly assess the longitudinal impact of $\mathrm{MC}$ on cognition and clinical variables in "real world" $\mathrm{MC}$ patients, and to quantify THC and CBD exposure. On average, $\mathrm{MC}$ patients in the current study reported higher CBD exposure relative to $\mathrm{THC}$ at all follow-up visits following initiation of MC use. As higher amounts of THC are often linked to cognitive decrements in recreational cannabis users (Kowal et al., 2015; Morgan et al., 2012; Ramaekers et al., 2006), and CBD has demonstrated efficacy in mitigating or preventing THC-related negative effects on cognition (Morgan et al., 2012; Solowij et al., 2018), this pattern of cannabinoid exposure may have influenced results. However, current findings also demonstrate that neither THC nor CBD use was directly correlated with observed improvements on measures of executive function. Rather, increased CBD exposure was associated with improved mood and anxiety symptoms. Correlation analyses suggest that improved cognitive performance (particularly faster Stroop Interference time) was associated with improvements in clinical symptoms. Accordingly, improvements in cognition may not be directly attributed to MC; instead MC treatment may indirectly improve cognition secondary to clinical improvements. In fact, symptoms commonly endorsed by $\mathrm{MC}$ patients, including anxiety and pain (the two most common indications reported by study participants), have been associated with reduced cognitive performance (Moriarty et al., 2011; Vytal et al., 2013). Although larger sample sizes are needed to perform mediation analyses, the current study provides preliminary evidence that clinical symptom improvement may result in improved performance, as patients may think more clearly if they feel better overall. Additionally, while the current study quantified THC and CBD exposure, other cannabinoids in patients' products may have also impacted study findings. As laboratory analyses of products quantified twelve cannabinoids, future studies will explore the relative contributions of additional constituents.

Further, it is imperative to assess age as a potential moderating variable. Unlike most studies of recreational cannabis users which assess young adults, who often initiate use during adolescence, those enrolled in the current study were significantly older (average age of 49). This is an important distinction, both because of neuromaturational changes during adolescence, and because marked changes occur in the endocannabinoid system in older relative to younger individuals. For example, preclinical studies reveal decreased levels of the endocannabinoid 2-arachidonoylglycerol (2-AG) in the aging mouse brain, and report that $\mathrm{CB} 1$ receptor binding peaks in puberty, remains stable early to mid-adulthood, and ultimately declines in older adulthood (Piyanova et al., 2015). Human studies have similarly revealed higher CB1 receptor binding in younger individuals relative to older adults (Di Marzo, Stella, \& Zimmer, 2015). In addition, although few studies have examined cognition secondary to $\mathrm{MC}$ use in older adults, several animal studies highlight the potential for cannabis to improve cognition in this population (Weinstein \& Sznitman, 2020), including one study demonstrating that administration of low-dose THC reversed age-related decline in older adult mice (Bilkei-Gorzo et al., 2017)

While results from the current study are promising, findings must be considered given several limitations. Although the demographic makeup of the current sample reflects Massachusetts, this sample of patients has limited diversity; thus, findings may not be generalizable to all populations. In future, larger analyses, a more diverse sample will facilitate examination of the potential influence of sex and race.

It is also of note that MC patients were examined over time but were not directly compared to patients with similar conditions who do not use cannabis. Accordingly, we have enrolled treatment-as-usual (TAU) group; once this sample is large enough, cognitive and clinical changes will be examined within and between both groups.

Another limitation is the potential confounding effects of treatment expectancy or the degree to which patients believe cannabis use can positively impact symptoms. Although no measures currently exist to assess treatment expectancies specifically related MC use, to begin to address this issue, patients completed the modified Marijuana Effect Expectancy Questionnaire-Brief (MEEQ-B; (Torrealday et al., 2008), which assesses positive and negative 
expectancies related to cannabis use in general. Interestingly, neither positive nor negative cannabis expectancies at baseline correlated with cognitive changes from baseline to 3 months of treatment, suggesting that cognitive and clinical improvements are not directly related to patients' preconceived beliefs regarding cannabis use. Further, as cognitive measures are objective, validated assessments, it is unlikely that MC patients would actually perform better simply as a result of perceived improvements.

Additionally, study results were not corrected for multiple comparisons; instead, we utilized a targeted approach by pre-selecting only the most relevant variables to limit the number of comparisons made, as done in previous studies (Fontes et al., 2011). This method avoids an overly conservative approach (e.g., Bonferroni corrections), which has the potential to increase the risk of Type II errors; results should therefore be considered accordingly.

Finally, the contribution of practice effects should be considered. However, utilizing alternate forms (for all tasks except Stroop and WCST) combined with the 3-6 month period between task administrations reduces the likelihood of practice effects. Studies examining practice effects reported no impact on LNS or Trail Making tasks, even with weekly administration (Beglinger et al., 2005), and studies noting practice effects on the Stroop typically utilized daily to weekly administration (Gul \& Humphreys, 2015). Further, in the current study, patients exhibited patterns of improvement on tasks of executive function but consistent, stable performance on tasks of memory, providing additional evidence that study findings are not likely attributable to practice effects. Future inclusion of a TAU group will further address this concern.

\section{CONCLUSIONS}

In a 12-month longitudinal, observational study, patients using MC for various medical conditions exhibited improved executive function and stable verbal learning and memory within the context of improvements on measures of mood, anxiety, and sleep relative to baseline. While greater improvement of clinical state over time was significantly associated with increased CBD exposure (mg/week), improved cognitive performance over time did not correlate with MC use. Future investigations examining the impact of individual cannabinoids and age of onset of use are warranted to clarify the implications of MC use. Ultimately, for MC patients, it is imperative to understand the relationship between these variables in order to maximize the therapeutic potential of cannabis while minimizing potential risk and harms.

\section{FUNDING}

This work was supported by private donations to the Marijuana Investigations for Neuroscientific Discovery (MIND) program at McLean Hospital.

\section{CONFLICTS OF INTEREST}

All authors have no conflicts of interest to disclose.

\section{REFERENCES}

Abrams, D.I. (2016). Integrating cannabis into clinical cancer care. Current Oncolology, 23(2), S8-S14. doi: 10.3747/co.23.3099

Atella, V., Mortari, A.P., Kopinska, J., Belotti, F., Lapi, F., Cricelli, C., \& Fontana, L. (2019). Trends in age-related disease burden and healthcare utilization. Aging Cell, 18(1), e12861.

Beck, A.T. \& Steer, R.A. (1990). Manual for the Beck Anxiety Inventory. San Antonio, TX: Psychological Corporation.

Beck, A.T., Ward, C.H., Mendelson, M., Mock, J., \& Erbaugh, J. (1961). An inventory for measuring depression. Archives of General Psychiatry, 4, 561-571.

Beglinger, L.J., Gaydos, B., Tangphao-Daniels, O., Duff, K., Kareken, D.A., Crawford, J., ... Siemers, E.R. (2005). Practice effects and the use of alternate forms in serial neuropsychological testing. Archives of Clinical Neuropsychology, 20(4), 517-529. doi: 10.1016/j.acn.2004.12.003

Berg, E.A. (1948). A simple objective technique for measuring flexibility in thinking. The Journal of General Psychology, 39, 15-22. doi: 10.1080/00221309.1948.9918159

Bergamaschi, M.M., Queiroz, R.H., Chagas, M.H., de Oliveira, D.C., De Martinis, B.S., Kapczinski, F., .. Crippa, J.A. (2011). Cannabidiol reduces the anxiety induced by simulated public speaking in treatment-naive social phobia patients. Neuropsychopharmacology, 36(6), 1219-1226. doi: 10.1038/ npp. 2011.6

Bilkei-Gorzo, A., Albayram, O., Draffehn, A., Michel, K., Piyanova, A., Oppenheimer, H., ... Zimmer, A. (2017). A chronic low dose of Delta(9)-tetrahydrocannabinol (THC) restores cognitive function in old mice. Nature Medicine, 23(6), 782-787. doi: 10.1038/nm.4311

Black, N., Stockings, E., Campbell, G., Tran, L.T., Zagic, D., Hall, W.D., .. . Degenhardt, L. (2019). Cannabinoids for the treatment of mental disorders and symptoms of mental disorders: A systematic review and meta-analysis. The Lancet Psychiatry, 6(12), 995-1010. doi: 10.1016/s2215-0366(19)30401-8

Bonaccorso, S., Ricciardi, A., Zangani, C., Chiappini, S., \& Schifano, F. (2019). Cannabidiol (CBD) use in psychiatric disorders: A systematic review. Neurotoxicology, 74, 282-298. doi: 10.1016/j.neuro.2019.08.002

Bouso, J.C., Jimenez-Garrido, D., Ona, G., Woznica, D., Dos Santos, R.G., Hallak, J.E.C., ... Farre, M. (2020). Quality of life, mental health, personality and patterns of use in self-medicated cannabis users with chronic diseases: A 12-month longitudinal study. Phytotherapy Research, 34(7), 1670-1677. doi: 10.1002/ptr.6639

Buysse, D.J., Reynolds, C.F., Monk, T.H., Berman, S.R., \& Kupfer, D.J. (1989). The pittsburgh sleep quailty index: A new instrument for psychiatric practice and research. Psychiatry Research, 28, 193-213.

Casey, B.J., Galvan, A., \& Hare, T.A. (2005). Changes in cerebral functional organization during cognitive development. Current Opinion in Neurobiology, 15(2), 239-244. doi: 10.1016/j.conb. 2005.03.012

Crean, R.D., Crane, N.A., \& Mason, B.J. (2011). An evidence based review of acute and long-term effects of cannabis use on executive cognitive functions. Journal of Addiction Medicine, 5(1), 1-8. doi: 10.1097/ADM.0b013e31820c23fa 
Crippa, J.A., Derenusson, G.N., Ferrari, T.B., Wichert-Ana, L., Duran, F.L., Martin-Santos, R., ... Hallak, J.E. (2011). Neural basis of anxiolytic effects of cannabidiol (CBD) in generalized social anxiety disorder: A preliminary report. Journal of Psychopharmacology, 25(1), 121-130. doi: 10.1177/02698811 10379283

De Vita, M.J., Moskal, D., Maisto, S.A., \& Ansell, E.B. (2018). Association of cannabinoid administration with experimental pain in healthy adults: A systematic review and meta-analysis. JAMA Psychiatry, 75(11), 1118-1127. doi: 10.1001/jama psychiatry.2018.2503

Desrosiers, N.A., Ramaekers, J.G., Chauchard, E., Gorelick, D.A., \& Huestis, M.A. (2015). Smoked cannabis' psychomotor and neurocognitive effects in occasional and frequent smokers. Journal of Analytical Toxicology, 39(4), 251-261. doi: 10.1093/ jat/bkv012

Di Marzo, V., Stella, N., \& Zimmer, A. (2015). Endocannabinoid signalling and the deteriorating brain. Nature Reviews Neuroscience, 16(1), 30-42. doi: 10.1038/nrn3876

Fontes, M.A., Bolla, K.I., Cunha, P.J., Almeida, P.P., Jungerman, F., Laranjeira, R.R., ... Lacerda, A.L. (2011). Cannabis use before age 15 and subsequent executive functioning. British Journal of Psychiatry, 198(6), 442-447. doi: 10.1192/bjp.bp.110.077479

Gallily, R., Yekhtin, Z., \& Hanuš, L.O. (2015). Overcoming the bell-shaped dose-response of cannabidiol by using cannabis extract enriched in cannabidiol. Pharmacology \& Pharmacy, 06(02), 75-85. doi: 10.4236/pp.2015.62010

Giedd, J.N., Blumenthal, J., Jeffries, N.O., Castellanos, F.X., Liu, H., Zijdenbos, A., . . Rapoport, J.L. (1999). Brain development during childhood and adolescence: A longitudinal MRI study. Nature Neuroscience, 2(10), 861-863. doi: 10.1038/13158

Gogtay, N., Nugent, T. F., 3rd, Herman, D. H., Ordonez, A., Greenstein, D., Hayashi, K.M., ... Thompson, P.M. (2006). Dynamic mapping of normal human hippocampal development. Hippocampus, 16(8), 664-672. doi: 10.1002/hipo.20193

Grella, C.E., Rodriguez, L., \& Kim, T. (2014). Patterns of medical marijuana use among individuals sampled from medical marijuana dispensaries in Los Angeles. Journal of Psychoactive Drugs, 46(4), 267-275. doi: 10.1080/02791072. 2014.944960

Gruber, S.A. \& Sagar, K.A. (2017). Marijuana on the mind? The impact of Marijuana on cognition, brain structure, and brain function, and related public policy implications. Behavioral and Brain Sciences, 4(1), 104-111. doi: 10.1177/2372732216684851

Gruber, S.A., Sagar, K.A., Dahlgren, M.K., Gonenc, A., Smith, R.T., Lambros, A.M., ... Lukas, S.E. (2018). The grass might be greener: Medical Marijuana patients exhibit altered brain activity and improved executive function after 3 months of treatment. Frontiers in Pharmacology, 8, 983. doi: 10.3389/fphar.2017. 00983

Gruber, S.A., Sagar, K.A., Dahlgren, M.K., Racine, M.T., Smith, R.T., \& Lukas, S.E. (2016). Splendor in the grass? A pilot study assessing the impact of medical marijuana on executive function. Frontiers in Pharmacology, 7, 355. doi: 10.3389/fphar.2016. 00355

Gul, A. \& Humphreys, G. (2015). Practice and colour-word integration in Stroop interference. Psicologica: International Journal of Methodology and Experimental Psychology, 36(1), 37-67.

Hart, C.L., Ilan, A.B., Gevins, A., Gunderson, E.W., Role, K., Colley, J., \& Foltin, R.W. (2010). Neurophysiological and cognitive effects of smoked marijuana in frequent users.
Pharmacol Biochem Behav, 96(3), 333-341. doi: 10.1016/j. pbb.2010.06.003

Hart, C.L., van Gorp, W., Haney, M., Foltin, R.W., \& Fischman, M.W. (2001). Effects of acute smoked marijuana on complex cognitive performance. Neuropsychopharmacology, 25(5), 757-765. doi: 10.1016/S0893-133X(01)00273-1

Heaton, R.K. \& Staff, P.A.R. Wisconsin Card Sorting Test: Computer Version 4. https://www.parinc.com/Products/Pkey/483

Houston, S.M., Herting, M.M., \& Sowell, E.R. (2014). The neurobiology of childhood structural brain development: Conception through adulthood. Current Topics in Behavioral Neuroscience, 16,3-17. doi: 10.1007/7854_2013_265

Hunault, C.C., Bocker, K.B., Stellato, R.K., Kenemans, J.L., de Vries, I., \& Meulenbelt, J. (2014). Acute subjective effects after smoking joints containing up to $69 \mathrm{mg}$ Delta9-tetrahydrocannabinol in recreational users: A randomized, crossover clinical trial. Psychopharmacology (Berl), 231(24), 4723-4733. doi: 10. 1007/s00213-014-3630-2

Ilgen, M.A., Bohnert, K., Kleinberg, F., Jannausch, M., Bohnert, A.S., Walton, M., \& Blow, F.C. (2013). Characteristics of adults seeking medical marijuana certification. Drug Alcohol Dependence, 132(3), 654-659. doi: 10.1016/j.drugalcdep.2013. 04.019

Jacobus, J. \& Tapert, S.F. (2014). Effects of cannabis on the adolescent brain. Current Pharmaceutical Design, 20(13), 2186-2193.

Kowal, M.A., Hazekamp, A., Colzato, L.S., van Steenbergen, H., van der Wee, N.J., Durieux, J., ... Hommel, B. (2015). Cannabis and creativity: Highly potent cannabis impairs divergent thinking in regular cannabis users. Psychopharmacology (Berl), 232(6), 1123-1134. doi: 10.1007/s00213-014-3749-1

Kuhathasan, N., Dufort, A., MacKillop, J., Gottschalk, R., Minuzzi, L., \& Frey, B.N. (2019). The use of cannabinoids for sleep: A critical review on clinical trials. Experimental Clinical Psychopharmacology, 27(4), 383-401. doi: 10.1037/pha000 0285

Lebel, C. \& Deoni, S. (2018). The development of brain white matter microstructure. Neuroimage, 182, 207-218. doi: 10.1016/j.neuro image.2017.12.097

Lezak, M.D., Howieson, D.B., Bigler, E.D., \& Tranel, D. (2012). Neuropsychological Assessment, (5th ed.). New York, NY: Oxford University Press.

Lisdahl, K.M., Gilbart, E.R., Wright, N.E., \& Shollenbarger, S. (2013). Dare to delay? The impacts of adolescent alcohol and marijuana use onset on cognition, brain structure, and function. Front Psychiatry, 4, 53. doi: 10.3389/fpsyt.2013.00053

Lisdahl, K.M., Wright, N.E., Kirchner-Medina, C., Maple, K.E., \& Shollenbarger, S. (2014). Considering Cannabis: The effects of regular cannabis use on neurocognition in adolescents and young adults. Current Addiction Reports, 1(2), 144-156. doi: 10.1007/ s40429-014-0019-6

Lorenzetti, V., Solowij, N., \& Yucel, M. (2016). The role of Cannabinoids in neuroanatomic alterations in cannabis users. Biological Psychiatry, 79(7), e17-e31. doi: 10.1016/j.biopsych. 2015.11.013

MacLeod, C.M. (1991). Half a century of research on the Stroop effect: An integrative review. Psychology Bulletin, 109(2), 163-203. doi: 10.1037/0033-2909.109.2.163

Martin-Santos, R., Crippa, J.A., Batalla, A., Bhattacharyya, S., Atakan, Z., Borgwardt, S., ... McGuire, P.K. (2012). Acute effects of a single, oral dose of d9-tetrahydrocannabinol (THC) and cannabidiol (CBD) administration in healthy volunteers. 
Current Pharmaceutical Design, 18(32), 4966-4979. doi: 10. 2174/138161212802884780

Masataka, N. (2019). Anxiolytic effects of repeated Cannabidiol treatment in teenagers with social anxiety disorders. Frontiers in Psychology, 10, 2466. doi: 10.3389/fpsyg.2019.02466

Millar, S.A., Stone, N.L., Bellman, Z.D., Yates, A.S., England, T.J., \& O'Sullivan, S.E. (2019). A systematic review of cannabidiol dosing in clinical populations. British Journal of Clinical Pharmacology, 85(9), 1888-1900. doi: 10.1111/bcp.14038

Minakawa, E.N., Wada, K., \& Nagai, Y. (2019). Sleep disturbance as a potential modifiable risk factor for Alzheimer's disease. International Journal of Molecular Science, 20(4), E803. doi: 10.3390/ijms20040803

Morgan, C.J., Gardener, C., Schafer, G., Swan, S., Demarchi, C., Freeman, T.P., ... Curran, H.V. (2012). Sub-chronic impact of cannabinoids in street cannabis on cognition, psychotic-like symptoms and psychological well-being. Psychology Medicine, 42(2), 391-400. doi: 10.1017/S0033291711001322

Morgan, C.J., Schafer, G., Freeman, T.P., \& Curran, H.V. (2010). Impact of cannabidiol on the acute memory and psychotomimetic effects of smoked cannabis: Naturalistic study: naturalistic study [corrected]. British Journal of Psychiatry, 197(4), 285-290. doi: 10.1192/bjp.bp.110.077503

Moriarty, O., McGuire, B.E., \& Finn, D.P. (2011). The effect of pain on cognitive function: A review of clinical and preclinical research. Progress in Neurobiology, 93(3), 385-404. doi: 10. 1016/j.pneurobio.2011.01.002

NASEM. (2017). The Health Effects of Cannabis and Cannabinoids: The Current State of Evidence and Recommendations for Research. Washington, DC.

Nunberg, H., Kilmer, B., Pacula, R.L., \& Burgdorf, J. (2011). An analysis of applicants presenting to a medical marijuana specialty practice in California. Journal of Drug Policy Analysis, 4(1), 1. doi: 10.2202/1941-2851.1017

Olla, P., Rykulski, N., Hurtubise, J.L., Bartol, S., Foote, R., Cutler, L., . . Erdodi, L.A. (2019). Short-term effects of cannabis consumption on cognitive performance in medical cannabis patients. Applied Neuropsychology Adult, 1-11. doi: 10.1080/ 23279095.2019.1681424

Park, J.Y. \& Wu, L.T. (2017). Prevalence, reasons, perceived effects, and correlates of medical marijuana use: A review. Drug Alcohol Dependence, 177, 1-13. doi: 10.1016/j.drugalc dep.2017.03.009

Pawasarat, I.M., Schultz, E.M., Frisby, J.C., Mehta, S., Angelo, M.A., Hardy, S.S., \& Kim, T.W.B. (2020). The efficacy of medical marijuana in the treatment of cancer-related pain. Journal of Palliative Medicine, 23(6), 809-816. doi: 10.1089/ jpm.2019.0374

Piyanova, A., Lomazzo, E., Bindila, L., Lerner, R., Albayram, O., Ruhl, T., ... Bilkei-Gorzo, A. (2015). Age-related changes in the endocannabinoid system in the mouse hippocampus. Mechanisms of Ageing and Development, 150, 55-64. doi: 10.1016/j.mad.2015.08.005

Poli, P., Crestani, F., Salvadori, C., Valenti, I., \& Sannino, C. (2018). Medical cannabis in patients with chronic pain: Effect on pain relief, pain disability, and psychological aspects. a prospective non randomized single arm clinical trial. Clinical Therapeutics, 169(3), e102-e107. doi: 10.7417/T.2018.2062

Pollock, V., Cho, D.W., Reker, D., \& Volavka, J. (1979). Profile of mood states: The factors and their physiological correlates. Journal of Nervous and Mental Disease, 167, 612-614.
Ramaekers, J.G., Kauert, G., van Ruitenbeek, P., Theunissen, E.L., Schneider, E., \& Moeller, M.R. (2006). High-potency marijuana impairs executive function and inhibitory motor control. Neuropsychopharmacology, 31(10), 2296-2303. doi: 10.1038/ sj.npp. 1301068

Robinson, S.M., Sobell, L.C., Sobell, M.B., \& Leo, G.I. (2014). Reliability of the timeline followback for cocaine, cannabis, and cigarette use. Psychology of Addictive Behaviors, 28(1), 154-162. doi: 10.1037/a0030992

Russo, E.B. (2007). History of cannabis and its preparations in saga, science, and sobriquet. Chemistry \& Biodiversity, 4(8), 1614-1648.

Ryan-Ibarra, S., Induni, M., \& Ewing, D. (2015). Prevalence of medical marijuana use in California, 2012. Drug Alcohol Reviews, 34(2), 141-146. doi: 10.1111/dar.12207

Sagar, K.A. \& Gruber, S.A. (2018). Marijuana matters: reviewing the impact of marijuana on cognition, brain structure and function, \& exploring policy implications and barriers to research. International Review of Psychiatry, 30(3), 1-17. doi: 10.1080/ 09540261.2018.1460334

Schmidt, M. (2016). Rey Auditory Verbal Learning Test Torrance. CA: Western Psychological Services.

Schubart, C.D., Sommer, I.E., van Gastel, W.A., Goetgebuer, R.L., Kahn, R.S., \& Boks, M.P. (2011). Cannabis with high cannabidiol content is associated with fewer psychotic experiences. Schizophrenia Research, 130(1-3), 216-221. doi: 10.1016/j. schres.2011.04.017

Schwartz, R.H., Gruenewald, P.J., Klitzner, M., \& Fedio, P. (1989). Short-term memory impairment in cannabis-dependent adolescents. The American Journal of Diseases of Children, 143(10), 1214-1219. doi: 10.1001/archpedi.1989.02150220110030

Scullin, M.K. \& Bliwise, D.L. (2015). Sleep, cognition, and normal aging: Integrating a half century of multidisciplinary research. Perspective Psychological on Science, 10(1), 97-137. doi: 10.1177/1745691614556680

Shannon, S., Lewis, N., Lee, H., \& Hughes, S. (2019). Cannabidiol in Anxiety and Sleep: A Large Case Series. The Permanente Journal, 23, 18-41. doi: 10.7812/TPP/18-041

Shi, L., Chen, S.J., Ma, M.Y., Bao, Y.P., Han, Y., Wang, Y.M., .. . Lu, L. (2018). Sleep disturbances increase the risk of dementia: A systematic review and meta-analysis. Sleep Medicine Reviews, 40, 4-16. doi: 10.1016/j.smrv.2017.06.010

Solowij, N., Broyd, S.J., Beale, C., Prick, J., Greenwood, L., van Hell, H.H., ... Yucel, M. (2018). Therapeutic effects of prolonged cannabidiol treatment on psychological symptoms and cognitive function in regular cannabis users: A pragmatic open-label clinical trial. Cannabis Cannabinoid Research, 3(1), 21-34. doi: 10.1089/can.2017.0043

Solowij, N., Jones, K.A., Rozman, M.E., Davis, S.M., Ciarrochi, J., Heaven, P.C., .. Yucel, M. (2011). Verbal learning and memory in adolescent cannabis users, alcohol users and non-users. Psychopharmacology (Berl), 216(1), 131-144. doi: 10.1007/ s00213-011-2203-X

Spielberger, C., Gorsuch, R., Lushene, P., Vagg, P., \& Jacobs, A. (1983). Manual for the State-Trait Anxiety Inventory. Palo Alto, CA: Consulting Psychologists Press.

Strauss, E., Sherman, E.M.S., \& Spreen, O. (2006). A Compendium of Neuropsychological Tests, (3rd ed.). New York, NY: Oxford University Press.

Substance Abuse and Mental Health Services Administration. (2019). Key Substance Use and Mental Health Indicators in 
the United States: Results from the 2018 National Survey on Drug Use and Health (HHS Publication No. PEP19-5068, NSDUH Series H-54). Rockville, MD.

Torrealday, O., Stein, L.A., Barnett, N., Golembeske, C., Lebeau, R., Colby, S.M., \& Monti, P.M. (2008). Validation of the marijuana effect expectancy questionnaire-brief. Journal of Children Adolescent Substance Abuse, 17(4), 1-17. doi: 10.1080/15470650802231861

Troutt, W.D. \& DiDonato, M.D. (2015). Medical cannabis in Arizona: Patient characteristics, perceptions, and impressions of medical cannabis legalization. Journal of Psychoactive Drugs, 47(4), 259-266. doi: 10.1080/02791072.2015.1074766

Vytal, K.E., Cornwell, B.R., Letkiewicz, A.M., Arkin, N.E., \& Grillon, C. (2013). The complex interaction between anxiety and cognition: insight from spatial and verbal working memory. Frontiers in Human Neuroscience, 7, 93. doi: 10.3389/fnhum. 2013.00093

Wachtel, S.R., ElSohly, M.A., Ross, S.A., Ambre, J., \& de Wit, H. (2002). Comparison of the subjective effects of Delta(9)tetrahydrocannabinol and marijuana in humans. Psychopharmacology (Berl), 161(4), 331-339. doi: 10.1007/ s00213-002-1033-2

Walsh, D., Nelson, K.A., \& Mahmoud, F.A. (2003). Established and potential therapeutic applications of cannabinoids in oncology. Support Care Cancer, 11(3), 137-143. doi: 10.1007/ s00520-002-0387-7

Wechsler, D. (1987). Manual for the Wechsler Memory ScaleRevised. San Antonio, TX: The Psychological Corporation.

Wechsler, D. (1999). Wechsler Abbreviated Scale of Intelligence. San Antonio, TX: The Psychological Corporation.

Weinstein, G. \& Sznitman, S.R. (2020). The implications of late-life cannabis use on brain health: A mapping review and implications for future research. Ageing Research Reviews, 59, 101041. doi: 10.1016/j.arr.2020.101041

Zeiger, J.S., Haberstick, B.C., Corley, R.P., Ehringer, M.A., Crowley, T.J., Hewitt, J.K., ... Rhee, S.H. (2010). Subjective effects to marijuana associated with marijuana use in community and clinical subjects. Drug Alcohol Dependence, 109(1-3), 161-166. doi: 10.1016/j.drugalcdep.2009.12.026

Zuardi, A.W., Cosme, R.A., Graeff, F.G., \& Guimaraes, F.S. (1993). Effects of ipsapirone and cannabidiol on human experimental anxiety. Journal of Psychopharmacology, 7(Suppl 1), 82-88. doi: 10.1177/026988119300700112

Zuardi, A.W., Shirakawa, I., Finkelfarb, E., \& Karniol, I.G. (1982). Action of cannabidiol on the anxiety and other effects produced by delta 9-THC in normal subjects. Psychopharmacology (Berl), 76(3), 245-250. 